\title{
The Impact of the Proposed Nigeria 330KV Integrated Network on the Transfer Capacity of the Existing 330KV Network
}

\author{
${ }^{1}$ Ogbikaya S.ogbikaya.stephen@edouniversity.edu.ng \\ ${ }^{2}$ Ike S.sam.ike@uniben.edu \\ ${ }^{3}$ Evbogbai M. J. E. evbogbaiedekin@edouniversity.edu.ng \\ ${ }^{1 \& 3}$ Department of Electrical and Electronic Engineering, Edo University Iyamho, Nigeria. \\ ${ }^{2}$ Department of Electrical and Electronic Engineering, University of Benin, Nigeria. \\ DOI: $10.31364 / \mathrm{SCIRJ} / \mathrm{v} 7.11 .2019 . P 0119607$ \\ http://dx.doi.org/10.31364/SCIRJ/v7.i1.2019.P0119607
}

All correspondence should be directed to Engr Ogbikaya S. (ogbikaya.stephen@edouniversity.edu.ng)

\begin{abstract}
The impact of the proposed integrated 330kV network on the transfer capacity of the existing 330kV network is analyzed in this work using ETAP 12.6 and the results analyzed with MATLAB software. The results obtained indicates that the transfer capacity of the entire network is increased by $2 \mathrm{GW}$ with the proposed integrated $330 \mathrm{kV}$ network. This will eradicate system collapse and more load will be accommodated in the network without stressing the network beyond it thermal limits.
\end{abstract}

Keywords: Integrated, Grid Network, Transfer Capacity, Voltage collapse, Generation, Transmission.

\section{INTRODUCTION}

The transfer capacity of a network is the maximum power in megawatt (MW) that can be transferred in the network. For a power system to be operated safely, the maximum power transferred must not exceed the transfer capacity of the network. When this is exceeded, the system experiences high power losses, frequency drop and voltage instability which will result to system load shedding, if not quickly carried out may led to voltage collapse on the entire network. Omorogiuwa and Ike (2014) in their study of Power flow control in the $330 \mathrm{KV}$ Integrated power network using unified power controller revealed that the Nigeria power system has a problem of insufficient transmission lines resulting to overloading and stressing of the network beyond their thermal limits as a result of increased load demand.

Ekeh and Evbogbai (2005) in their study of the NEPA Grid system failures in Nigeria revealed that the transmission disturbance contributed about $84.21 \%$ of the grid system failure. The report also identify the phase to earth faults as the most common case of the grid system disturbance.

Byung and Kwang (1991) reported that the heart of voltage stability problems occur when there is a voltage drop due to high load demand on the network causing voltage instability that leads to voltage collapse. This voltage collapse occurs when there is an initial slow progressive decline in the voltage magnitude of the power system bus finally rapid decline in the voltage magnitude. Ekeh and Evbogbai (2005) in their work suggested ways for improvement on the reliability of the grid system to include reinforcement of the network and transformer upgrading, routine maintenance culture of transmission equipment, overhauling and repairs of various electric power plants, installation of sophisticated and modern metering supervisory and control equipment at the National Control Centres, building of more generating station and reinforcement of grid system through dualization of single circuit. Abdullah (2009) in his work, stated that acceptable reliability level of power system requires additional generating capacity to meet the expected increase in future electrical load demands. The rapid increase in the demand for electricity is as a result of population growth, 
industrial expansion and increase in customer's appliances which have necessitated the stepping up of generation and transmission capacities of the grid network to deliver quality power supply. Onojo, Ononiwu and Okozi (2013) research shows that the existing grid networks is inadequate and lack the capacity to provide the quality and quantity of power for the country. This work present the impact of the proposed Nigeria $330 \mathrm{kV}$ integrated network on the transfer capacity of the existing $330 \mathrm{kV}$ network.

\section{MATERIALS AND METHOD}

The materials utilized in the analysis of this work include; ETAP 12.6 software, the Existing 330KV grid network, the Proposed $330 \mathrm{KV}$ grid network and MATLAB software for the analysis of results obtained.

In this work, the Existing $330 \mathrm{KV}$ and proposed integrated $330 \mathrm{KV}$ network of the Nigeria National grid were modelled in ETAP 12.6 environment as shown in figure 1 and figure 2 respectively.

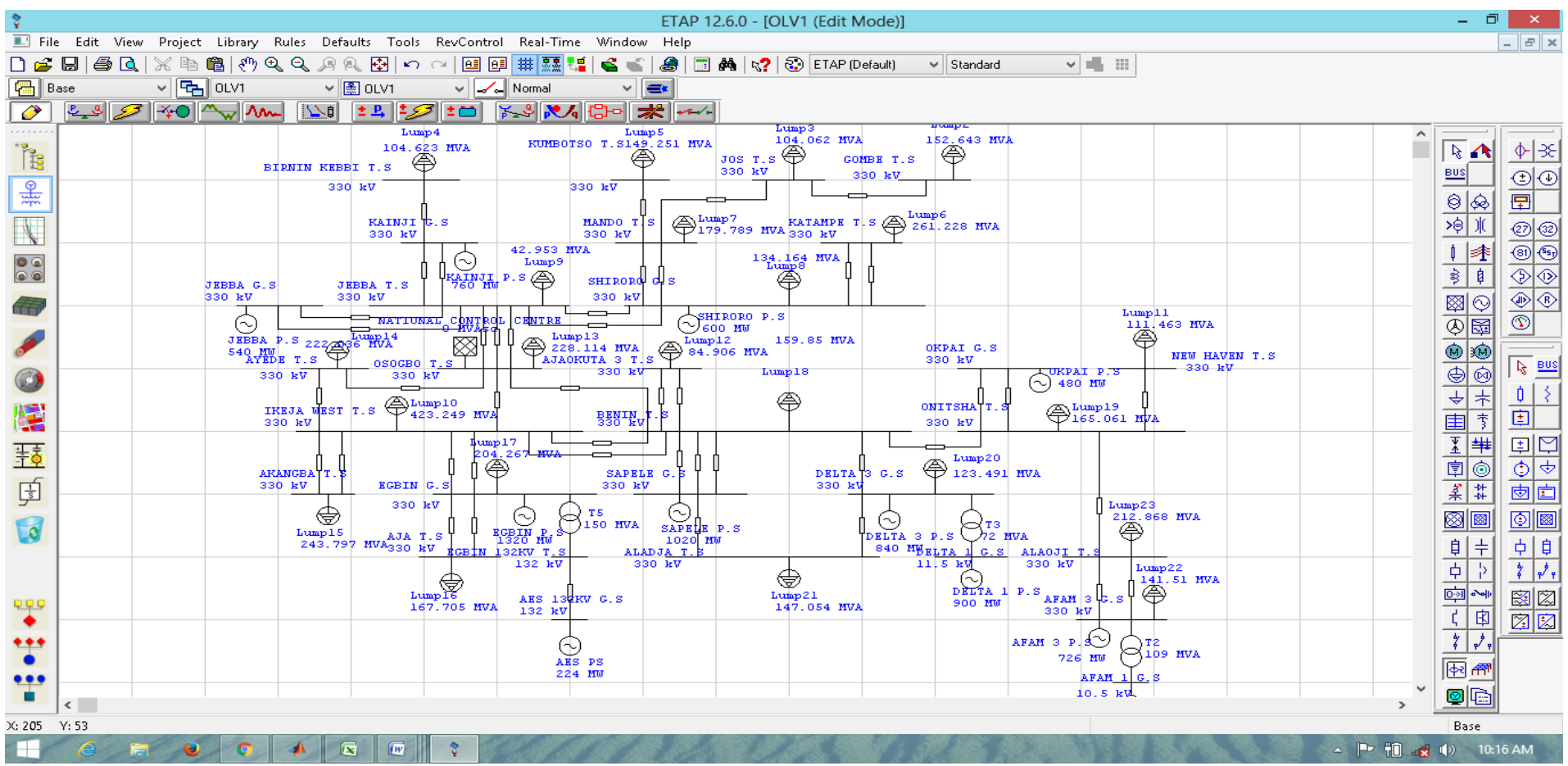

Figure 1: Model of Existing 330KV Network using ETAP 12.6 Software 


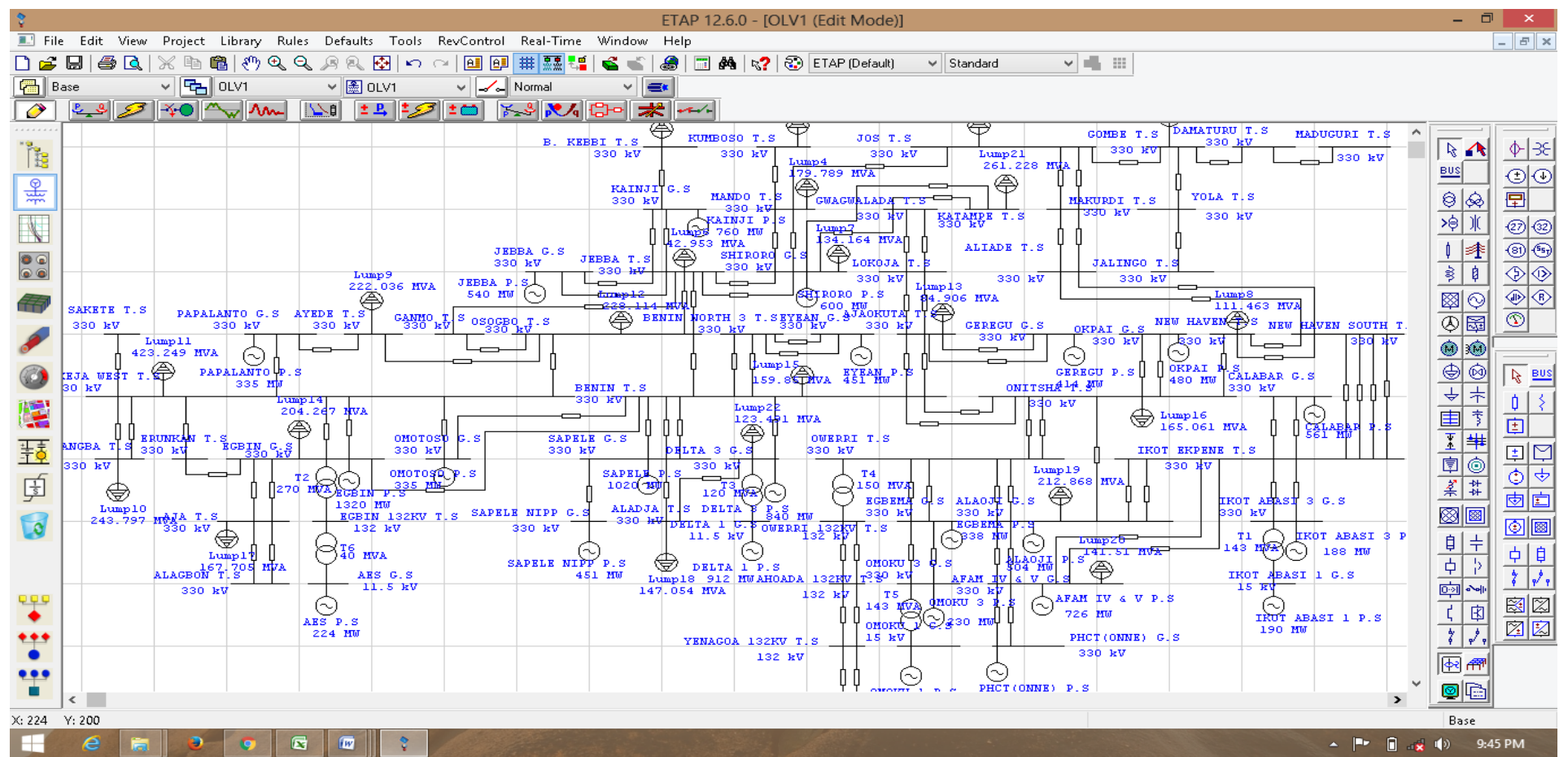

Figure 2: Model of Proposed Integrated 330KV Network using ETAP 12.6 Software

These models were simulated (run mode) in the same environment using Newton Raphson Iteration Algorithm with a precision of 0.0001 as a result of it easy convergence as shown in figure 3 and figure 4 .

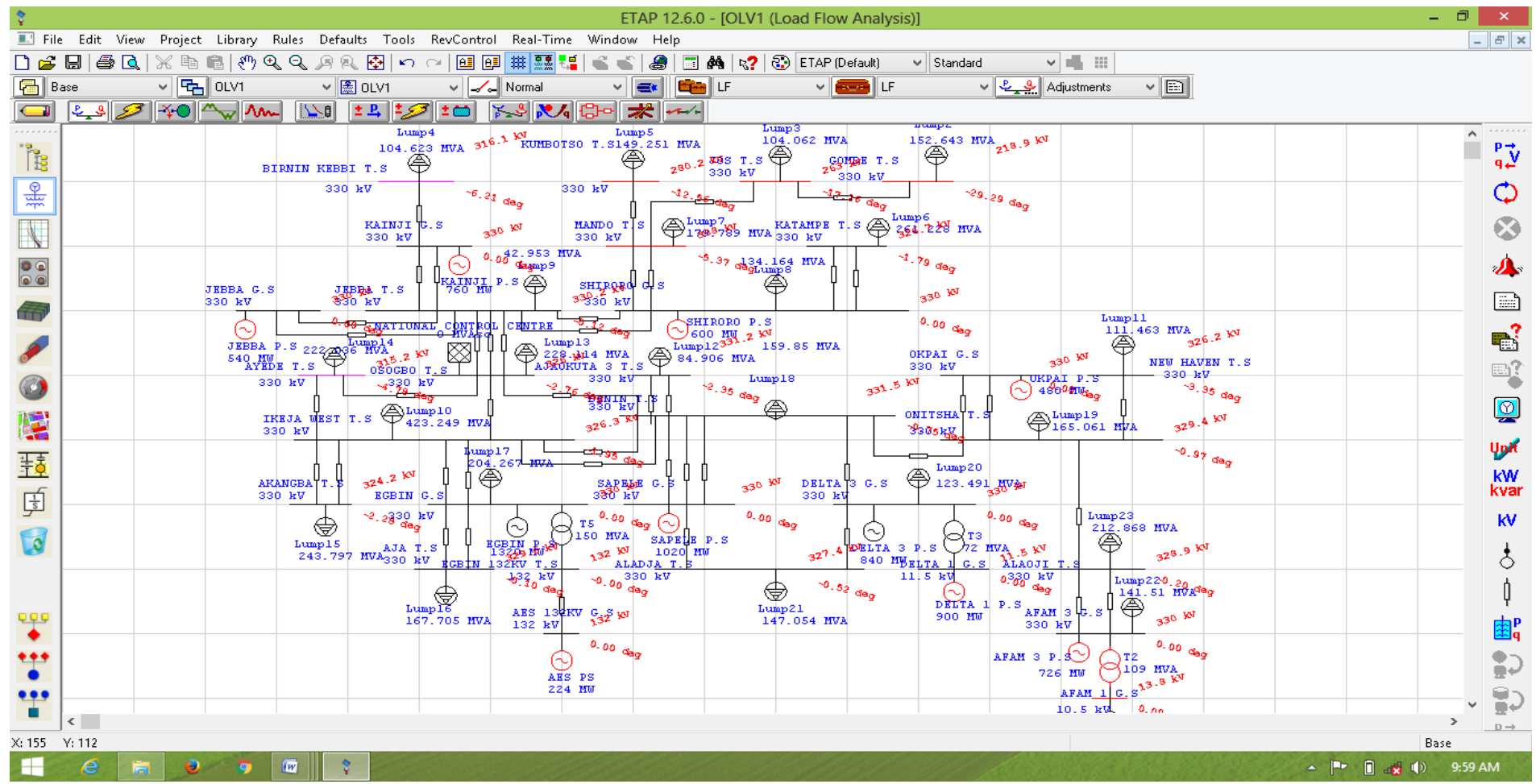

Figure 3: Simulation (Run Mode) of Existing 330KV Network using ETAP 12.6 Software 


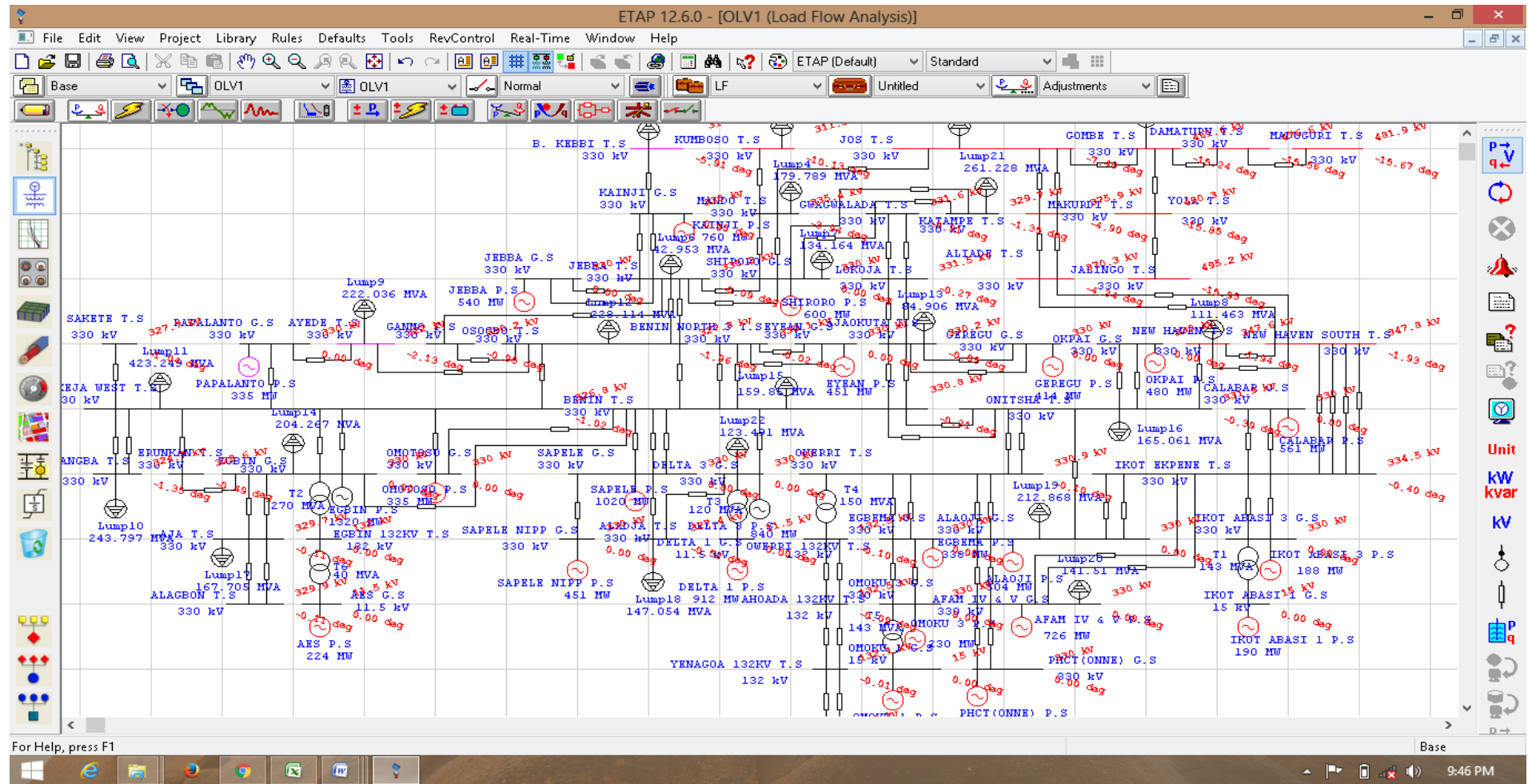

Figure 4: Simulation (Run mode) of Proposed Integrated 330KV Network using ETAP 12.6 Software

\section{RESULT AND DISCUSSION}

The simulated and calculated value of the transfer capacity of both the existing $330 \mathrm{KV}$ and proposed integrated $330 \mathrm{KV}$ network is tabulated in table 1 and table 2 with their respective graphs obtained from MATLAB.

Table 1: Transfer Capacity of the Existing 330KV Network

\begin{tabular}{|c|l|l|c|c|}
\hline $\begin{array}{c}\text { Transmis } \\
\text { sion } \\
\text { Lines } \\
(330 \mathrm{KV})\end{array}$ & From Bus & To Bus & $\begin{array}{c}\text { Simulated Transfer } \\
\text { Capacity of the } \\
\text { lines from Power } \\
\text { Flow Result (MW) }\end{array}$ & $\begin{array}{c}\text { Calculated value of } \\
\text { Maximum Power } \\
\text { Transferred on each } \\
\text { lines (MW) }\end{array}$ \\
\hline 1 & GOMBE T.S & JOS T.S & 122.06 & 75.03 \\
\hline 2 & KUMBOTSO T.S & MANDO T.S & 118.97 & 65.12 \\
\hline 3 & $\begin{array}{l}\text { BIRNIN KEBBI } \\
\text { T.S }\end{array}$ & KAINJI G.S & 87.54 & 87.77 \\
\hline 4 & KAINJI G.S & JEBBA T.S & 5.95 & 22.93 \\
\hline 5 & KAINJI G.S & JEBBA T.S & 5.95 & 22.93 \\
\hline 6 & MANDO T.S & $\begin{array}{l}\text { SHIRORO } \\
\text { G.S }\end{array}$ & 256.78 & 27.18 \\
\hline
\end{tabular}




\begin{tabular}{|c|c|c|c|c|}
\hline 7 & JOS T.S & MANDO T.S & 223.85 & 55.78 \\
\hline 8 & KATAMPE T.S & $\begin{array}{l}\text { SHIRORO } \\
\text { G.S }\end{array}$ & 117.53 & 86.25 \\
\hline 9 & JEBBA T.S & JEBBA G.S & 125.37 & 4.79 \\
\hline 10 & JEBBA T.S & JEBBA G.S & 125.37 & 4.79 \\
\hline 11 & JEBBA T.S & $\begin{array}{l}\text { SHIRORO } \\
\text { G.S }\end{array}$ & 1.98 & 69.09 \\
\hline 12 & JEBBA T.S & $\begin{array}{l}\text { SHIRORO } \\
\text { G.S } \\
\end{array}$ & 1.98 & 69.09 \\
\hline 13 & JEBBA T.S & $\begin{array}{l}\text { OSOGBO } \\
\text { T.S }\end{array}$ & 75.21 & 44.45 \\
\hline 14 & JEBBA T.S & $\begin{array}{l}\text { OSOGBO } \\
\text { T.S }\end{array}$ & 75.21 & 44.45 \\
\hline 15 & NEW HAVEN T.S & $\begin{array}{l}\text { ONITSHA } \\
\text { T.S }\end{array}$ & 109.49 & 27.18 \\
\hline 16 & OKPAI G.S & $\begin{array}{l}\text { ONITSHA } \\
\text { T.S }\end{array}$ & 112.07 & 47.92 \\
\hline 17 & OKPAI G.S & $\begin{array}{l}\text { ONITSHA } \\
\text { T.S } \\
\end{array}$ & 112.07 & 47.92 \\
\hline 18 & AJAOKUTA 3 T.S & BENIN T.S & 36.23 & 55.21 \\
\hline 19 & AJAOKUTA 3 T.S & BENIN T.S & 36.27 & 55.21 \\
\hline 20 & BENIN T.S & $\begin{array}{l}\text { OSOGBO } \\
\text { T.S } \\
\end{array}$ & 37.19 & 71.07 \\
\hline 21 & OSOGBO T.S & AYEDE T.S & 82.79 & 32.56 \\
\hline 22 & OSOGBO T.S & $\begin{array}{l}\text { IKEJA } \\
\text { WEST T.S }\end{array}$ & 13.9 & 71.35 \\
\hline 23 & IKEJA WEST T.S & $\begin{array}{l}\text { AKANGBA } \\
\text { T.S }\end{array}$ & 93.84 & 5.10 \\
\hline 24 & IKEJA WEST T.S & $\begin{array}{l}\text { AKANGBA } \\
\text { T.S } \\
\end{array}$ & 93.84 & 5.10 \\
\hline 25 & EGBIN G.S & AJA T.S & 74.96 & 8.39 \\
\hline 26 & EGBIN G.S & AJA T.S & 74.96 & 8.39 \\
\hline 27 & IKEJA WEST T.S & EGBIN G.S & 296.12 & 37.13 \\
\hline 28 & IKEJA WEST T.S & EGBIN G.S & 296.12 & 37.13 \\
\hline 29 & BENIN T.S & SAPELE G.S & 61.12 & 14.16 \\
\hline 30 & BENIN T.S & SAPELE G.S & 61.12 & 14.16 \\
\hline 31 & BENIN T.S & SAPELE G.S & 61.12 & 14.16 \\
\hline 32 & SAPELE G.S & ALADJA T.S & 40.3 & 17.84 \\
\hline 33 & DELTA 3 G.S & ALADJA T.S & 79.33 & 9.06 \\
\hline 34 & BENIN T.S & $\begin{array}{l}\text { DELTA } 3 \\
\text { G.S } \\
\end{array}$ & 28.56 & 30.30 \\
\hline 35 & ONITSHA T.S & BENIN T.S & 9.18 & 38.79 \\
\hline 36 & ONITSHA T.S & BENIN T.S & 9.18 & 38.79 \\
\hline 37 & AJAOKUTA 3 T.S & BENIN T.S & 36.23 & 55.21 \\
\hline 38 & ONITSHA T.S & ALAOJI T.S & 23.61 & 39.07 \\
\hline
\end{tabular}



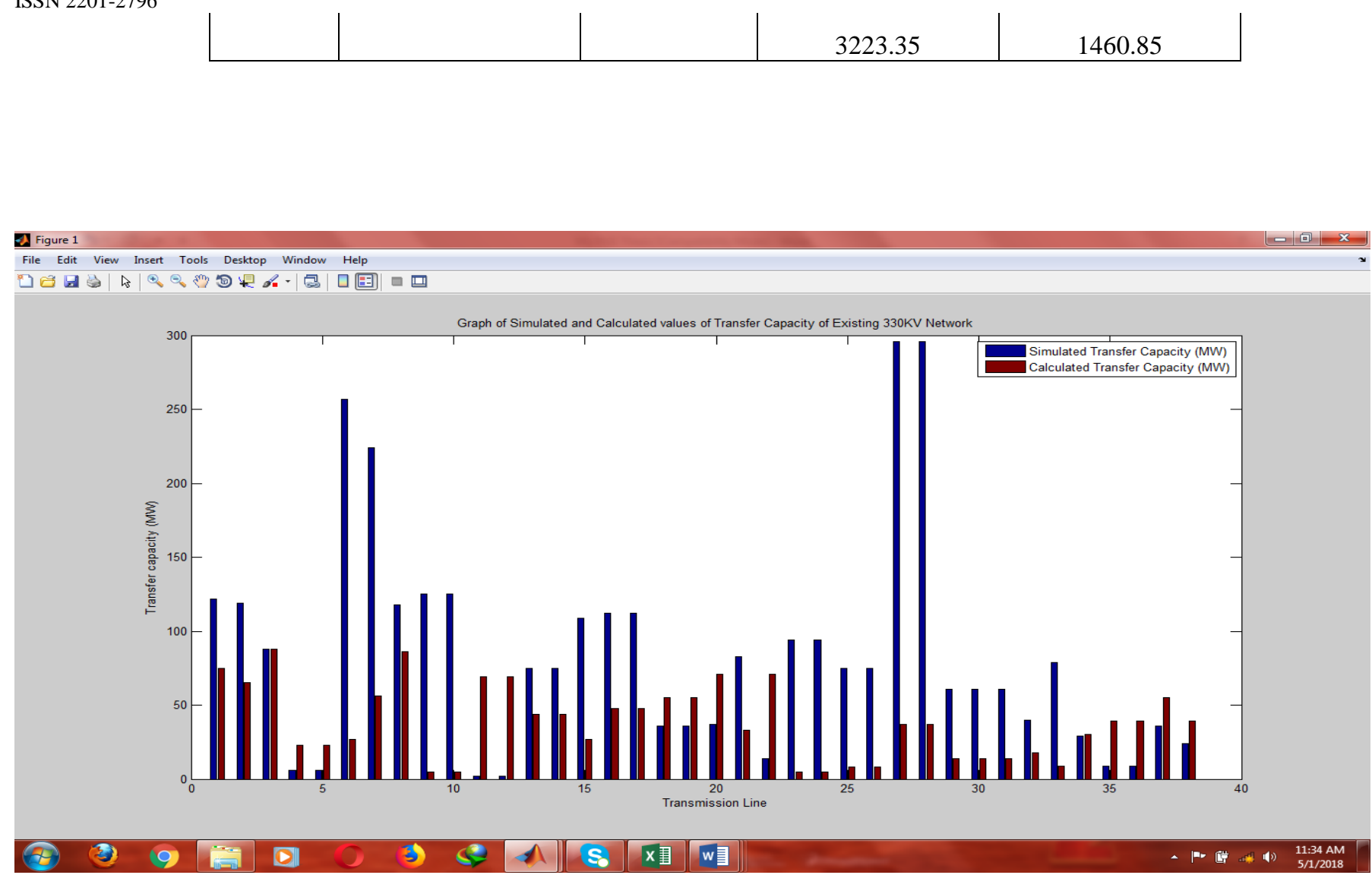

Figure 5: Graph of Simulated and Calculated values of the Transfer Capacity of the Existing 330KV Network. 
Table 2: Transfer Capacity of the Proposed Integrated 330KV Network

\begin{tabular}{|c|c|c|c|c|}
\hline $\begin{array}{c}\text { Transmission } \\
\text { Lines } \\
(330 \mathrm{KV})\end{array}$ & From Bus & To Bus & $\begin{array}{l}\text { Simulated } \\
\text { Transfer } \\
\text { Capacity of } \\
\text { the lines from } \\
\text { Power Flow } \\
\text { Result (MW) }\end{array}$ & $\begin{array}{l}\text { Calculated } \\
\text { value of } \\
\text { Maximum } \\
\text { Power } \\
\text { Transferred on } \\
\text { each lines } \\
\text { (MW) }\end{array}$ \\
\hline 1 & GOMBE T.S & JOS T.S & 170.121 & 75.03 \\
\hline 2 & DAMATURU T.S & GOMBE T.S & 0.755 & 38.22 \\
\hline 3 & MADUGURI T.S & DAMATURU T.S & 0.081 & 39.64 \\
\hline 4 & KUMBOSO T.S & MANDO T.S & 123.223 & 65.12 \\
\hline 5 & B. KEBBI T.S & KAINJI G.S & 83.653 & 87.77 \\
\hline 6 & KAINJI G.S & JEBBA T.S & 4.455 & 22.93 \\
\hline 7 & KAINJI G.S & JEBBA T.S & 4.455 & 22.93 \\
\hline 8 & MANDO T.S & SHIRORO G.S & 173.974 & 27.18 \\
\hline 9 & MANDO T.S & SHIRORO G.S & 173.974 & 27.18 \\
\hline 10 & MAKURDI T.S & ALIADE T.S & 115.528 & 29.95 \\
\hline 11 & MAKURDI T.S & ALIADE T.S & 115.528 & 29.95 \\
\hline 12 & YOLA T.S & JALINGO T.S & 0.071 & 37.37 \\
\hline 13 & JOS T.S & MANDO T.S & 56.1 & 55.78 \\
\hline 14 & KATAMPE T.S & $\begin{array}{l}\text { GWAGWALADA } \\
\text { T.S }\end{array}$ & 150.996 & 8.49 \\
\hline 15 & KATAMPE T.S & SHIRORO G.S & 85.277 & 86.25 \\
\hline 16 & $\begin{array}{l}\text { GWAGWALADA } \\
\text { T.S }\end{array}$ & SHIRORO G.S & 68.41 & 68.28 \\
\hline 17 & $\begin{array}{l}\text { GWAGWALADA } \\
\text { T.S }\end{array}$ & LOKOJA T.S & 41.637 & 83.85 \\
\hline 18 & $\begin{array}{l}\text { GWAGWALADA } \\
\text { T.S }\end{array}$ & LOKOJA T.S & 41.637 & 83.85 \\
\hline 19 & JOS T.S & MAKURDI T.S & 114.681 & 137.76 \\
\hline 20 & JOS T.S & MAKURDI T.S & 114.681 & 137.76 \\
\hline 21 & GOMBE T.S & YOLA T.S & 1.609 & 61.44 \\
\hline 22 & JEBBA T.S & JEBBA G.S & 93.659 & 4.79 \\
\hline 23 & JEBBA T.S & JEBBA G.S & 93.659 & 4.79 \\
\hline 24 & SHIRORO G.S & JEBBA T.S & 1.479 & 69.09 \\
\hline 25 & SHIRORO G.S & JEBBA T.S & 1.479 & 69.09 \\
\hline 26 & JEBBA T.S & OSOGBO T.S & 53.002 & 44.45 \\
\hline 27 & JEBBA T.S & OSOGBO T.S & 53.002 & 44.45 \\
\hline 28 & JEBBA T.S & GANMO T.S & 53.492 & 19.82 \\
\hline 29 & LOKOJA T.S & AJAOKUTA T.S & 41.708 & 22.76 \\
\hline 30 & LOKOJA T.S & AJAOKUTA T.S & 41.708 & 22.76 \\
\hline 31 & ALIADE T.S & $\begin{array}{l}\text { NEW HAVEN } \\
\text { SOUTH T.S }\end{array}$ & 119.649 & 89.84 \\
\hline 32 & $\begin{array}{l}\text { NEW HAVEN } \\
\text { SOUTH T.S }\end{array}$ & ALIADE T.S & 119.649 & 89.84 \\
\hline 33 & $\begin{array}{l}\text { NEW HAVEN } \\
\text { SOUTH T.S }\end{array}$ & $\begin{array}{l}\text { NEW HAVEN } \\
\text { T.S }\end{array}$ & 30.439 & 2.99 \\
\hline 34 & NEW HAVEN & NEW HAVEN & 30.439 & 2.99 \\
\hline
\end{tabular}




\begin{tabular}{|c|c|c|c|c|}
\hline & SOUTH T.S & T.S & & \\
\hline 35 & $\begin{array}{l}\text { NEW HAVEN } \\
\text { T.S }\end{array}$ & ONITSHA T.S & 52.881 & 27.18 \\
\hline 36 & CALABAR G.S & $\begin{array}{l}\text { IKOT EKPENE } \\
\text { T.S }\end{array}$ & 33.232 & 42.12 \\
\hline 37 & CALABAR G.S & $\begin{array}{l}\text { IKOT EKPENE } \\
\text { T.S }\end{array}$ & 33.232 & 42.12 \\
\hline 38 & $\begin{array}{l}\text { NEW HAVEN } \\
\text { SOUTH T.S }\end{array}$ & $\begin{array}{l}\text { IKOT EKPENE } \\
\text { T.S }\end{array}$ & 76.632 & 85.65 \\
\hline 39 & $\begin{array}{l}\text { NEW HAVEN } \\
\text { SOUTH T.S }\end{array}$ & $\begin{array}{l}\text { IKOT EKPENE } \\
\text { T.S }\end{array}$ & 76.632 & 85.65 \\
\hline 40 & $\begin{array}{l}\text { NEW HAVEN } \\
\text { SOUTH T.S }\end{array}$ & $\begin{array}{l}\text { IKOT EKPENE } \\
\text { T.S }\end{array}$ & 76.632 & 85.65 \\
\hline 41 & $\begin{array}{l}\text { NEW HAVEN } \\
\text { SOUTH T.S }\end{array}$ & $\begin{array}{l}\text { IKOT EKPENE } \\
\text { T.S }\end{array}$ & 76.632 & 85.65 \\
\hline 42 & SAKETE T.S & IKEJA WEST T.S & 0.005 & 19.82 \\
\hline 43 & $\begin{array}{l}\text { PAPALANTO } \\
\text { G.S }\end{array}$ & IKEJA WEST T.S & 158.38 & 8.49 \\
\hline 44 & AYEDE T.S & $\begin{array}{l}\text { PAPALANTO } \\
\text { G.S }\end{array}$ & 165.827 & 16.99 \\
\hline 45 & OKPAI G.S & ONITSHA T.S & 37.849 & 47.92 \\
\hline 46 & OKPAI G.S & ONITSHA T.S & 37.849 & 47.92 \\
\hline 47 & GEREGU G.S & AJAOKUTA T.S & 80.84 & 29.95 \\
\hline 48 & GEREGU G.S & AJAOKUTA T.S & 80.84 & 29.95 \\
\hline 49 & AJAOKUTA T.S & BENIN T.S & 3.1 & 55.21 \\
\hline 50 & AJAOKUTA T.S & BENIN T.S & 3.1 & 55.21 \\
\hline 51 & EYEAN G.S & $\begin{array}{l}\text { BENIN NORTH } 3 \\
\text { T.S }\end{array}$ & 35.684 & 2.99 \\
\hline 52 & EYEAN G.S & $\begin{array}{l}\text { BENIN NORTH } 3 \\
\text { T.S }\end{array}$ & 35.684 & 2.99 \\
\hline 53 & $\begin{array}{l}\text { BENIN NORTH } 3 \\
\text { T.S }\end{array}$ & BENIN T.S & 35.68 & 5.66 \\
\hline 54 & $\begin{array}{l}\text { BENIN NORTH } 3 \\
\text { T.S }\end{array}$ & BENIN T.S & 35.68 & 5.66 \\
\hline 55 & OSOGBO T.S & BENIN T.S & 31.483 & 71.07 \\
\hline 56 & OSOGBO T.S & GANMO T.S & 53.177 & 24.63 \\
\hline 57 & OSOGBO T.S & AYEDE T.S & 12.452 & 32.56 \\
\hline 58 & OSOGBO T.S & IKEJA WEST T.S & 15.444 & 71.35 \\
\hline 59 & $\begin{array}{l}\text { IKOT EKPENE } \\
\text { T.S }\end{array}$ & $\begin{array}{l}\text { IKOT ABASI } 3 \\
\text { G.S }\end{array}$ & 31.903 & 44.92 \\
\hline 60 & $\begin{array}{l}\text { IKOT EKPENE } \\
\text { T.S }\end{array}$ & $\begin{array}{l}\text { IKOT ABASI } 3 \\
\text { G.S }\end{array}$ & 31.903 & 44.92 \\
\hline 61 & IKEJA WEST T.S & AKANGBA T.S & 93.897 & 5.1 \\
\hline 62 & IKEJA WEST T.S & AKANGBA T.S & 93.897 & 5.1 \\
\hline 63 & IKEJA WEST T.S & ERUNKAN T.S & 76.673 & 9.06 \\
\hline 64 & EGBIN G.S & ERUNKAN T.S & 76.822 & 8.49 \\
\hline 65 & EGBIN G.S & AJA T.S & 74.976 & 8.39 \\
\hline 66 & EGBIN G.S & AJA T.S & 74.976 & 8.39 \\
\hline 67 & AJA T.S & ALAGBON T.S & 0.001 & 7.36 \\
\hline 68 & AJA T.S & ALAGBON T.S & 0.001 & 7.36 \\
\hline 69 & IKEJA WEST T.S & EGBIN G.S & 160.691 & 37.13 \\
\hline 70 & IKEJA WEST T.S & EGBIN G.S & 160.691 & 37.13 \\
\hline 71 & IKEJA WEST T.S & OMOTOSO G.S & 29.696 & 45.3 \\
\hline 72 & BENIN T.S & OMOTOSO G.S & 6.666 & 33.98 \\
\hline 73 & BENIN T.S & EGBIN G.S & 3.669 & 61.72 \\
\hline 74 & BENIN T.S & SAPELE G.S & 33.137 & 14.16 \\
\hline
\end{tabular}




\begin{tabular}{|c|l|l|c|c|}
\hline 75 & BENIN T.S & SAPELE G.S & 33.137 & 14.16 \\
\hline 76 & BENIN T.S & SAPELE G.S & 15.997 & 14.16 \\
\hline 77 & SAPELE G.S & ALADJA T.S & 40.296 & 17.84 \\
\hline 78 & DELTA 3 G.S & ALADJA T.S & 79.333 & 9.06 \\
\hline 79 & BENIN T.S & DELTA 3 G.S & 7.475 & 30.3 \\
\hline 80 & ONITSHA T.S & BENIN T.S & 4.843 & 38.79 \\
\hline 81 & ONITSHA T.S & BENIN T.S & 4.843 & 38.79 \\
\hline 83 & ONITSHA T.S & OWERRI T.S & 31.896 & 44.92 \\
\hline 84 & ONITSHA T.S & OWERRI T.S & 31.896 & 44.92 \\
\hline 85 & OWERRI T.S & EGBEMA G.S & 21.284 & 8.49 \\
\hline 86 & ONITSHA T.S & ALBEMA G.S & 21.284 & 8.49 \\
\hline 87 & OWERRI T.S & ALAOJI G.S & 50.465 & 16.99 \\
\hline 88 & OWERRI T.S & ALAOJI G.S & 10.642 & 16.99 \\
\hline 89 & IKOT EKPENE & ALAOJI G.S & 62.965 & 16.99 \\
\hline 90 & $\begin{array}{l}\text { TKOT EKPENE } \\
\text { T.S }\end{array}$ & ALAOJI G.S & 62.965 & 22.76 \\
\hline 91 & $\begin{array}{l}\text { IKOT EKPENE } \\
\text { T.S }\end{array}$ & $\begin{array}{l}\text { AFAM IV \& V } \\
\text { G.S }\end{array}$ & 26.585 & 22.76 \\
\hline 92 & $\begin{array}{l}\text { AFAM IV \& V } \\
\text { G.S }\end{array}$ & $\begin{array}{l}\text { IKOT EKPENE } \\
\text { T.S }\end{array}$ & 26.585 & 53.91 \\
\hline & & & 5115.889 & 3549.52 \\
\hline
\end{tabular}

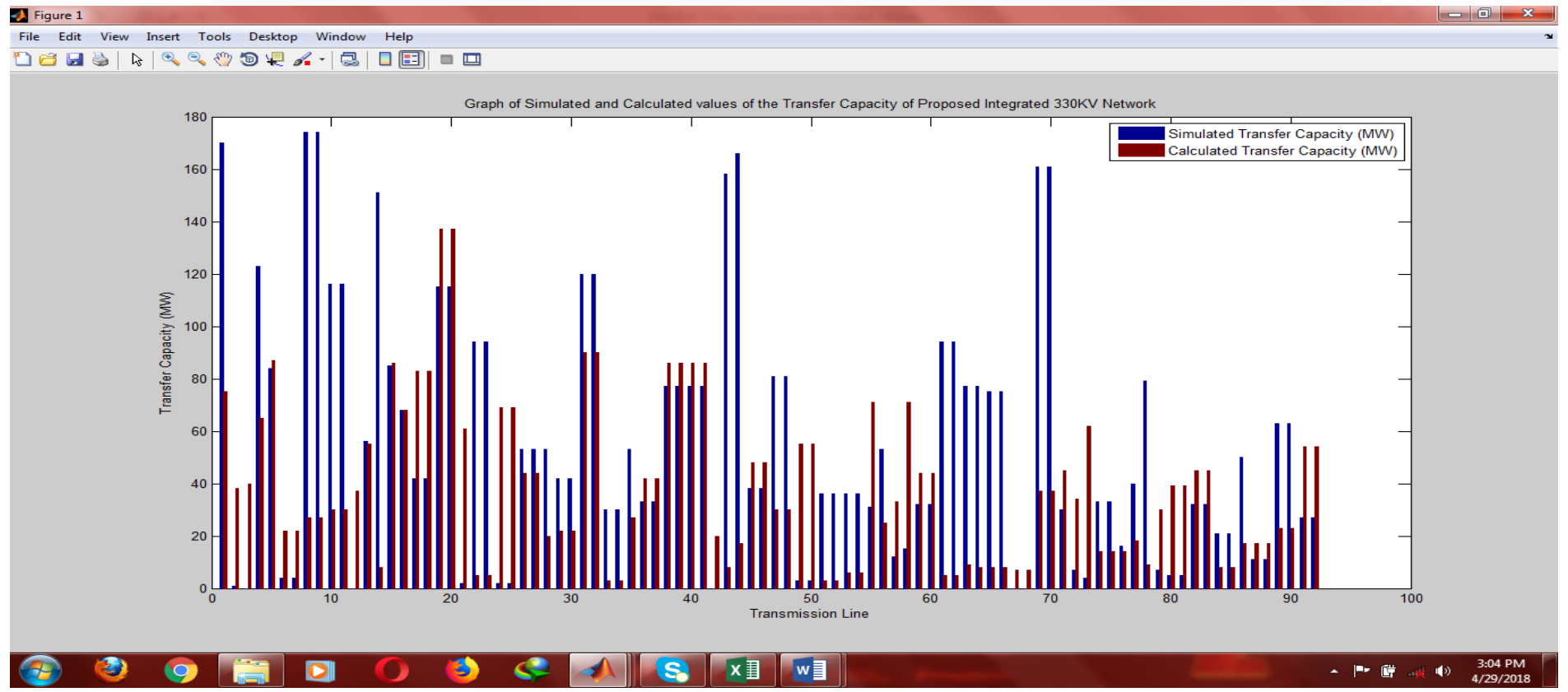

Figure 6: Graph of Simulated and Calculated values of the Transfer Capacity of the Proposed Integrated 330KV Network 
Table 3: Summary of Simulated and Calculated Transfer Capacity of Existing 330KV network and Proposed Integrated 330KV network

\begin{tabular}{|l|c|c|}
\hline Network Type & $\begin{array}{c}\text { Simulated Transfer } \\
\text { Capacity (MW) }\end{array}$ & $\begin{array}{c}\text { Calculated Transfer } \\
\text { Capacity (MW) }\end{array}$ \\
\hline $\begin{array}{l}\text { Proposed Integrated } \\
\text { 330KV Network }\end{array}$ & $5,115.89$ & $3,549.52$ \\
\hline $\begin{array}{l}\text { Existing 330KV } \\
\text { Network }\end{array}$ & $3,223.35$ & $1,460.85$ \\
\hline Difference & $1,892.54$ & $2,088.67$ \\
\hline
\end{tabular}

From the analysis in table 3 , the transfer capacity of the existing $330 \mathrm{KV}$ network is improved by approximately $2 \mathrm{GW}$ either by simulation or by calculation

\section{CONCLUSION}

The study reveal that the transfer capacity of the entire network is increased by $2 \mathrm{GW}$ with the proposed integrated $330 \mathrm{KV}$ network. This will help the system to accommodate more load and reduce load shedding drastically and also eradicate system collapses in the network as result of voltage instability.

\section{REFERENCES}

Abdullah M. Al-Shaalan (2009).’Problems associated with power system planning in developing countries", the online journal on Electronics and Electrical Engineering, Vol.1, No.1, Pages 43-48.

Byung Ha Lee \& Kwang Y. Lee (1991). “A study on voltage collapse mechanism in electric power systems”, Transactions on power systems, Vol. 6, Pages 966 - 974, August.

Ekeh J. C \& Evbogbai M. J. E. (2005). "A study of the NEPA Grid system failure in Nigeria”, Journal of Applied and Basic Sciences, Vol. 3(1 \& 2), Pages $157-165$.

Omoroguiwa E. \& Ike S. (2014). "Power flow control in the Nigeria 330KV integrated power network using unified power flow controller", International Journal of Engineering Innovation and Research, Vol.3, Issue 6, Pages 724 - 731, October.

Onojo, O. J., Ononiwu, G. C. \& Okozi,S. O. (2013). “Analysis of power flow of Nigerian 330kv grid system (pre and post) using Matlab", European Journal of Natural and Applied Sciences, Vol. 1, Iss (2), Pages 59-66. 
Scientific Research Journal (SCIRJ), Volume VII, Issue I, January 2019 